\title{
Favoriser le développement de la conscience épistémologique des étudiants lors de l'approprition de l'écrit glottodidactique en français et en polonais. Le cas des étudiants polonais d'ethnolinguistique
}

\author{
Stimulating the development of students' \\ epistemological consciousness in the acquisition \\ of glottodidactic discourse in French and Polish: \\ The case of Polish ethnolinguistics students
}

\begin{abstract}
The paper is a reflection on the development of academic literacy skills in French and Polish while writing a diploma paper in glottodidactics by Polish third year ethnolinguistics students. The first part of the paper focuses on a diploma paper in glottodidactics as an important component of philological education at Adam Mickiewicz University in Poznań. Next, the author analyses the concept of epistemological consciousness and its impact on the development of student autonomy during the acquisition of glottodidactic discourse and glottodidactic knowledge as a specialist knowledge which they gain. The author's conception, referring to writing research papers in line with the principles of glottodidactics as a research field, is based on the assumption that students should become aware of the epistemological specifics of glottodidactics and include them both in the form and the content of their diploma papers. Finally, the author discusses various stages in the development of students' epistemological consciousness and looks into the processes the supervisor needs to engage in for effective research supervision.
\end{abstract}

KEYWORDS: Epistemological consciousness, student autonomy, academic literacy skills, glottodidactic discourse, glottodidactic knowledge, glottodidactics as an academic discipline, diploma papers in glottodidactics.

MOTS-CLÉS: Conscience épistémologique, autonomisation des étudiants, littéracies universitaires, discours glottodidactique, savoirs glottodidactiques, glottodidactique comme discipline académique, mémoire de licence en glottodidactique. 


\section{QUELQUES REMARQUES PRÉALABLES}

L'initiation des étudiants à la rédaction du discours de recherche est l'une des visées de la formation académique au niveau de la licence à l'université polonaise. Or, il s'agit de l'appropriation du discours, mais aussi des savoirs qui $s^{\prime}$ incrivent dans le monde de recherche du domaine dans lequel les étudiants commencent à se spécialiser. Certes, au niveau de la licence, on ne s'attend pas encore à ce que le discours d'un étudiant puisse être considéré comme celui $d^{\prime}$ experts et nous admettons qu'il s'agit du discours dont l'auteur est un novice en matière de littéracie universitaire, ce qui ne nous empêche pas de reconnaître la dimension épistémologique dans le processus de la rédaction du mémoire de licence (désormais ML). Autrement dit, d'après nous, la rédaction du ML nécessite la réflexion et la prise de conscience de la part des étudiants en ce qui concerne la façon dont les connaissances, et en particulier les connaissances scientifiques, sont construites et diffusées. Ainsi, nous présupposons que l'enseignant responsable de l'accompagnement des étudiants dans leur parcours d'initiation à ces nouvelles pratiques discursives devrait favoriser le développement de leur conscience épistémologique et le présent article sera consacré au développement de la conscience épistémologique des étudiants polonais d'ethnolinguistique lors de la préparation de leur ML en didactologie des langues-cultures (désormais la DLC) ou plutôt en glottodidactique ${ }^{1}$, nom polonais de cette discipline académique.

Tout au long de notre texte, nous chercherons à démontrer pourquoi la conscience épistémologique des étudiants joue un rôle considérable dans leur appropriation du discours glottodidactique. De manière plus précise, dans un premier temps, nous caractériserons la spécificité du contexte académique dans lequel s'inscrit la rédaction du ML par nos étudiants. Puis, c'est la notion de conscience épistémologique qui sera définie, ceci par rapport à la spécificité épistémologique de la glottodidactique en tant que discipline académique. Enfin, dans la troisième partie de l'article, nous présenterons les différentes étapes dans le développement de la conscience épistémologique des étudiants pour démontrer par la suite quelles démarches formatives sont à appliquer par le tuteur du mémoire pour favoriser le développement de ce type de conscience dans le cadre des consultations avec ses étudiants.

${ }^{1}$ Dans le présent article, on utilise les termes didactologie des langues-cultures (cf. Galisson 1986, 1990) et glottodidactique comme synonymiques, tout en reconnaissant les dimensions culturelle et institutionnelle de ces deux appellations (Orchowska 2014a, 2017). Par conséquent, on traitera ici également les adjectifs didactologique et glottodidactique comme des synonymes. De même, on prendra en considération les dimensions culturelles et institutionnelles des pratiques de littéracies universitaires (cf. Delcambre \& Lahanier-Reuter 2012) par rapport à l'appropriation du discours glottodidactique dans le cadres des études philologiques en Pologne. 
Il faut souligner que la rédaction du ML constitue une étape particulière dans la formation des étudiants qui ont choisi la philologie comme filière à l'université polonaise. Parmi les étudiants de la Faculté des Langues et Littératures Modernes à l'Université Adam Mickiewicz de Poznań qui décident de consacrer leur troisième année de licence à la rédaction en français d'un mémoire portant sur les problèmes de recherche relevant de la glottodidactique, les plus nombreux sont ceux de philologie romane, mais un groupe à part est constitué également d'étudiants d'ethnolinguistique. Comme ces étudiants, à la différence de leurs homologues de l'Institut de philologie romane, n'ont pas la possibilité de suivre le séminaire de licence à proprement parlée, ils ne découvrent l'écrit de recherche dans leur domaine de spécialité qu'à travers la rédaction de leurs ML qui est encadrée par des consultations régulières avec leur tutrice de mémoire - glottodidacticienne et auteure du présent article ayant le statut de maître de conférences - et dans une certaine mesure, à travers un cours facultatif d'initiation à la DLC qui se déroule en français ou en polonais en fonction du niveau de connaissances linguistiques des étudiants intéressés par ce cours qui ne dure qu'un semestre (cf. annexe 1 avec le programme du cours). Il est à préciser que les étudiants en question peuvent rédiger leur ML en glottodidactique en français ou en polonais également en fonction de leur niveau de maitrise du français (cf. annexe 2 avec les titres originaux des ML en DLC rédigés et soutenus entre octobre 2012 et septembre 2019 dans l'ancien Institut de Linguistique à la Faculté des Langues et Littératures Modernes de l'Université Adam Mickiewicz de Poznań3 $)$. Ce cours facultatif permet également de développer leur littéracie glottodidactique en tant que variante de littéracies universitaires générales (cf. Chiss 2012 ; Delcambre \& Lahanier-Reuter 2012) par rapport à la compréhension écrite du discours glottodidactique, tout en privilégiant la communication orale des savoirs disciplinaires, par exemple, pendant les séquences de discussion auxquelles participent activement les étudiants.

${ }^{2} \mathrm{D}$ 'après la réglementation interne qui est en vigueur à l'Université Adama Mickiewicz de Poznań, y compris à la Faculté des Langues et Littératures Modernes, il faut au moins 7 étudiants inscrits au cours de séminaire de licence pour que ceci soit compris dans l'emploi du temps de l'enseignant-tuteur du ML. Sinon, celui-ci doit coopérer avec les étudiants seulement dans le cadre de ses heures de permanence.

${ }^{3} \mathrm{Il}$ est à préciser qu'à partir du $1^{\mathrm{er}}$ janvier 2020 l'ancien Institut de Linguistique à l'Université Adam Mickiewicz de Poznań fut transformé en trois unités de recherche et de formation : l'Institut de l'Ethnocommunication, la Chaire de la Méthodologie en recherche en Linguistique et la Section des Philologies Rares faisant désormais partie intégrante de l'Institut de Linguistique Appliquée. Toutes ces trois unités appartiennent au Département des Langues et Littératures Modernes de l'Université Adama Mickiewicz et on continue d'y proposer le cursus en ethnolinguistique au niveau de la licence et de la maîtrise. 
À notre sens, ce qui conditionne le succès de l'apprentissage de l'écrit glottodidactique par un(e) étudiant(e) est incontestablement le processus de son autonomisation dans le sens d'une prise de responsabilité de sa formation et de la gestion de son auto-apprentissage. Ainsi, dans le présent article, on se focalisera sur ce qui, selon nous, est crucial pour l'efficacité formative d'une modalité pédagogique particulière d'accompagnement des étudiants à l'entrée en écriture de recherche que sont les consultations entre ces étudiants et leur tutrice du ML et que l'on peut considérer comme une forme particulière d'une éducation personnalisée (cf. Karpińska-Musiał 2016) et autonome (cf. Wilczyńska 2002, 2004). Notre réflexion portera ici sur le rapport entre la conscience épistémologique des étudiants polonais en ethnolinguistique et leur appropriation de l'écrit et des savoirs glottodidactiques. En effet, nous avançons que le développement explicite de la conscience des scripteurs novices en matière de spécificité épistémologique du domaine de recherche dans lequel s'inscrivent les problèmes abordés dans leurs ML devrait constituer une partie intégrante de l'initation des étudiants au discours de recherche en DLC, ceci déjà au niveau de la licence et donc en troisième année de leurs études à l'université. Parallèlement, nous chercherons à expliquer quel est l'impact de cette prise de conscience sur la façon dont les étudiants s'approprient, interprètent et appliquent leurs connaissances didactologiques tant sur le plan de pratiques d'enseignement-apprentissage des langues et cultures que sur celui de la recherche.

Côté contexte éducatif dans lequel sont formés nos étudiants, il est à souligner que même si la rédaction du ML en glottodidactique constitue une composante importante de la formation philologique à l'Université Adam Mickiewicz de Poznań, seulement un nombre très restreint des étudiants d'ethnolinguistique choisit la DLC comme domaine de spécialité. Ainsi, les plus nombreux sont ceux qui rédigent leur ML en sciences du langage et ceux qui situent leur premier écrit de recherche dans le domaine de la traductologie. Sur ce plan, il faut souligner que ce qui distingue la rédaction du ML en glottodidactique des mémoires inscrits dans d'autres domaines de spécialité est incontestablement le fait que les étudiants doivent s'approprier les savoirs glottodidactiques de façon autonome, car leur cursus universitaire ne prévoit qu'un cours facultatif d'initiation à la DLC réalisé en seulement 30 heures, alors que les sciences du langage y sont beaucoup plus présentes ${ }^{4}$.

${ }^{4} \mathrm{D}^{\prime}$ après la nouvelle classification des disciplines scientifiques, qui est en vigueur en Pologne depuis le $1^{\text {er }}$ octobre 2019, la DLC n'est pas reconnue comme une science autonome et officiellement elle fait partie inégrante des sciences du langage. Et pourtant, un tel état des choses ne reflète pas l'ensemble des points de vue le statut scientifique de la glottodidactique que représentent de nombreux spécialistes polonais dans le domaine (cf. Zając 2010 ; Dakowska 2014, 2018 ; Orchowska en préparation). De plus, d'après nous, on devrait reconnaître la glottodidactique également comme 


\section{DE LA CONSCIENCE ÉPISTÉMOLOGIQUE DES ÉTUDIANTS À L'ÉTAPE DE LEUR INITIATION À L'ÉCRIT GLOTTODIDACTIQUE}

Comme nous nous focalisons ici sur la rédaction par les étudiants de leurs ML en DLC, nous tenons à mettre en valeur les liens entre l'initiation à l'écriture de recherche, le cas échéant l'initiation à la littéracie glottodidactique, et le développement des connaissances des étudiants en question dans leur discipline de spécialité à travers le recours au concept de conscience épistémologique du scripteur novice. Nous tenterons de développer ici l'idée d'après laquelle l'émergence de la conscience épistémologique chez les étudiants en licence conditionne la qualité de la rédaction de leurs écrits glottodidactiques d'initation, mais aussi leur façon de s'approprier des savoirs spécialisés dans le domaine concerné. Par conséquent, à notre sens, tout au long de l'accompagnement de ces étudiants lors de l'élaboration de leurs premiers écrits autonomes en DLC, on devrait, en tant qu'enseignant-tuteur du ML, les amener à prendre conscience de ce qu'est la spécificité épistémologique de la discipline académique à laquelle ils s'initient. Toutefois, l'enjeu est particulièrement difficile à définir et encore plus à relever au niveau de la licence, car c'est un diplôme de premier cycle. En effet, il s'agit d'une étape d'initiation des étudiants aussi bien à la glottodidactique comme discipline académique et comme domaine de recherche qu'à l'écrit glottodidactique, alors que dans la majorité ils n'ont au départ aucune expérience en matière de discours de recherche au sens large du terme de littéracies universitaires, que ce soit par rapport à la lecture ou à la rédaction de textes.

\subsection{Le niveau de la licence en ethnolinguistique comme étape d'initiation à la dimension discursive et épistémologique de la glottodidactique}

Les études d'ethnolinguistique à l'Université Adam Mickiewicz de Poznań constituent une filière particulière dans le cadre du cursus universitaire en philologie ${ }^{5}$. En Pologne, on a d'habitude des études philologiques focalisées sur une

une discipline académique et, par conséquent, nous voyons la nécessité de l'enseigner à l'université avec la prise en considération de sa spécificité épistémologique et méthodologique.

${ }^{5}$ Dans le contexte universitaire polonais, les disciplines académiques dans le sens des matières étant l'objet de la formation au niveau de l'enseignement supérieur ne se superposent pas toujours avec les noms des domaines de recherche. Ainsi, on parle des études de philologie, alors que la philologie ne fonctionne pas comme une discipline de recherche dans la Pologne contemporaine et les maîtres de conférences qui assurent les cours dans le cadre du cursus philologique se spécialisent en tant que chercheurs en DLC, linguistique, traductologie ou encore dans la théorie de la littérature. 
langue et la / les culture/s qui s'exprime(nt) à travers cette langue, telles les philologies allemande, anglaise, espagnole, italienne, romane (l'adjectif française serait dans ce dernier cas plus approprié), etc. Or les étudiants en ethnolinguistique choisissent deux langues étrangères : une première langue étrangère (à un niveau avancé) et une deuxième langue étrangère (à un niveau débutant) dans lesquelles ils se spécialisent et qu'ils étudient à travers les cours de langues (660 heures pour la première et 690 heures pour la deuxième réparties sur trois années d'études) et de traduction pratique (60 heures de traduction vers la première langue étrangère contre 30 vers la deuxième), le français ayant dans notre unité de formation le statut de deuxième langue étrangère.

Parallèlement, le programme d'études en ethnolinguistique au niveau de la licence comprend des matières à caractère savant telles que : l'introduction à la linguistique, l'introduction à la théorie de la littérature, l'introduction à l'ethnologie et l'anthropologie culturelle, la logique et l'informatique (en première année), la sociolinguistique, la philosophie, deux cours facultatifs dont l'un peut être le cours d'introduction à la DLC (en deuxième année d'études), la psycholinguistique et l'acquisition du langage, l'histoire et la culture de la deuxième langue étrangère, le séminaire de licence (en troisième année). De plus, lors du premier cycle d'études, les étudiants doivent faire un stage de quatre semaines dans un cadre professionnel lié à la pratique de la langue étrangère choisie.

Nous pouvons constater que le développement des compétences en littéracies universitaires ne constitue pas la priorité dans le cursus des étudiants d'ethnolinguistique au niveau de la licence. De plus, s'ils sont exposés aux discours disciplinaires de quelques domaines de recherche, il s'agit toujours d'une initiation à ce genre de discours et aux savoirs qui d'ailleurs sont transmis par les enseignants surtout à travers le discours oral. Il est à préciser également que l'appropriation des savoirs disciplinaires s'effectuent essentiellement en langue maternelle des étudiants, à part le cours facultatif d'introduction à la DLC qui peut se dérouler en français à condition toutefois que le nombre d'étudiants intéressés par ce cours dispensé en langue française ne soit pas inférieur à onze.

La spécificité du cursus en ethnolinguistique dans la perspective du développement de la littéracie glottodidactique étant décrite, il serait maintenant approprié de se demander comment accompagner les étudiants dans ce contexte formatif pour favoriser le développement de leurs compétences en littéracies universitaires, notamment pour ce qui est du discours didactologique. À notre sens, le processus de rédaction du ML en DLC nécessite que les étudiants s'appro-

Il est également à préciser qu'à partir de l'année universitaire 2019/2020, la Faculté des Langues et Littératures Modernes de l'Université Adam Mickiewicz de Poznań fait partie intégrante de l'unité de formation universitaire appelée l'École des Études sur la Langue et la Littérature. 
prient le discours et les savoirs dans cette discipline à un niveau qui leur permet de bien comprendre les textes spécialisés et d'être à même de rédiger leurs propres écrits de recherche, dans un souci de dialogue (inter)disciplinaire avec d'autres spécialistes, tout en confrontant les théories et modèles scientifiques du domaine avec la réalité empirique de l'enseignement-apprentissage des langues qui constitue l'objet de recherche en DLC.

Concernant les exigences formelles auxquelles doit correspondre le ML en DLC, W. Wilczyńska (1996) précisait par rapport aux ML préparés par les étudiants aux $\mathrm{NKJO}^{6} \mathrm{qu}^{\prime} \mathrm{il}$ ne s'agissait pas de décrire une situation ou un thème ni de présenter un compte rendu des publications sur un sujet ou produire un manuel, mais qu'il fallait plutôt traiter en profondeur un problème, tout en le rapportant à une situation didactique bien précise. Le processus d'élaboration du ML doit donc amener les étudiants à développer des savoir-faire tels que : savoir choisir, savoir contextualiser, savoir apprécier, savoir analyser avec un esprit critique, savoir communiquer, savoir argumenter, savoir échanger, etc. Ainsi, nous pouvons constater que la préparation du ML constitue une expérience intéressante et valorisante pour les étudiants tant sur le plan discursif que sur celui de la recherche. Il s'agit donc d'initier les étudiants à la recherche et au discours de recherche dans le domaine de leur spécialité qu'est la glottodidactique.

Cependant, il faut tenir compte du fait qu'à la différence des étudiants suivant le cours de séminaire en DLC à la philologie romane, anglaise, allemande, etc., les étudiants d'ethnolinguistique n'effectuent pas de stage pédagogique en milieu scolaire, car leur cursus ne comprend pas l'enseignement relevant des sciences de l'éducation et, par conséquent, ils ne sont pas habilités à faire ce type de stage. Tout au long de la préparation de leur ML en DLC, ils ne peuvent pas donc effectuer une recherche d'action. Ainsi, sont-ils obligés de recourir à d'autres types de recherche glottodidactique, comme par exemple l'étude des représentations des acteurs de l'enseignement-apprentissage des langues (et cultures) à travers des questionnaires ou enquêtes et des interviews guidées et semi-guidée, ou bien ils peuvent effectuer une analyse critique, comparative et / ou chronologique $\mathrm{du}$ contenu des dispositifs d'enseignement-apprentissage, tels que les manuels de langues ou les sites Internet à caractère didactique (cf. annexe 2).

${ }^{6}$ Les NKJO (pl. Nauczycielskie Kolegia Języków Obcych) étaient des centres (universitaires ou non) où on formait les futurs enseignants de langues vivantes en Pologne, mais ils ont été fermés (à part le KJO à l'Université de Varsovie qui a été transformé en Centre de Formation des Enseignants de Langues Étrangères et d'Éducation Européenne de l'Université de Varsovie). Aujourd'hui, ce sont donc les philologies fonctionnant à l'intérieur des universités dans le cadre des Facultés des Langues et Littératures Modernes qui s'occupent de la formation des futurs enseignants de langues. 


\subsection{La notion de conscience épistémologique par rapport à la spécificité épistémologique de la glottodidactique en tant que discipline académique}

Quant à la notion de conscience épistémologique d'un(e) étudiant(e) qui décide de se spécialiser en glottodidactique, nous la définissons comme une prise de conscience de la façon dont les connaissances du domaine de la DLC sont construites et diffusées, mais aussi comme une attitude particulière à l'égard de ces connaissances, qui se reflète notamment au niveau de l'appropriation de ces savoirs. Ainsi, nous postulons que les étudiants novices doivent d'une part, changer leurs représentations initiales relatives à l'enseignement-apprentissage des langues, car celles-ci relèvent avant tout de la soi-disant doxa et d'autre part, s'ouvrir à l'épstémè, en opérant la rupture épistémologique (Bachelard 1938 ; Orchowska 2010a, 2014b).

Plus précisément, nous reconnaissons que les étudiants d'ethnolinguistique pour être à même de se positionner dans le cadre de leur ML vis-à-vis des questions relatives au processus d'enseignement / apprentissage et à d'autres questions relatives à la DLC doivent comprendre comment se construisent des connaissances disciplinaires, quelle en est leur nature épistémologique et notamment leur lien avec les pratiques relevant du processus d'enseignementapprentissage des langues. Or, lors de l'étape d'initiation à la démarche épistémologique du domaine de la DLC, il ne s'agit pas encore, à notre sens, $d^{\prime}$ amener les étudiants à construire des connaissances scientifiques, mais de les aider à s'y référer afin qu'ils comprennent mieux le problème glottodidactique analysé, ce qui pourrait justement déboucher sur le développement d'une posture de chercheur novice. Et par la suite, cette posture peut se développer soit vers celle d'un enseignant de langue en tant que praticien réfléchi (cf. Schön 1983), soit vers celle d'un chercheur conscient en tant que membre autonome de la communauté scientifique (cf. Orchowska 2017), quoique les deux ne s'excluent pas nécessairement (cf. Karpińska-Musiał \& Orchowska 2014).

Parallèlement, étant donné que la conscience épistémologique des auteurs des ML en DLC est en lien direct avec leur compréhension de la façon dont les connaissances glottodidactiques sont construites et diffusées par les chercheurs, il est ici incontournable de se demander quelle est la nature des savoirs disciplinaires en DLC dont nos étudiants devraient prendre conscience. En effet, il faut tenir compte du fait que les conceptions de ce qu'est la DLC en tant que discipline de recherche sont très variées et qu'elles se différencient non seulement en fonction du contexte socio-culturel dans lequel fonctionnent les chercheurs 
(cf. Orchowska 2014a), mais aussi à l'intérieur du même pays, voire dans le même cadre universitaire (cf. Orchowska 2013a, 2013b).

À titre d'exemple, R. Galisson qualifie la DLC de discipline praxéologique. Autrement dit, selon lui, ce domaine de recherche n'a pas pour finalité la théorie, la connaissance pour la connaissance, mais "l'action et plus précisément l'optimisation du processus de transmission des connaissances relatives à l'éducation aux langues (et aux cultures) » (Galisson \& Puren 1999 : 82). Ainsi, dans les étapes relatives à la construction des connaissances dans le domaine, on part du terrain par l'intermédiaire de l'observation de l'objet d'étude pour par la suite s'en écarter par la problématisation en contexte et la conceptualisation, et on finit par le retour sur le terrain en proposant une intervention en tant que solution par rapport aux problèmes analysés. Nous retrouvons une conception similaire de la DLC dans les discours didactologique de la spécialiste polonaise en FLE J. Zając (2010). D'autre part, pour deux autres glottodidacticiennes polonaises : M. Dakowska (2010) et W. Wilczyńska (2010) aussi bien que pour le chercheur français J.-L. Chiss (2010), la visée principale de la DLC est de nature cognitive et non praxéologique. Ainsi, en DLC les recherches fondamentales devraient du point de vue de ces trois chercheurs et bien d'autres (cf. Orchowska 2020) précéder les recherches appliquées focalisées sur l'optimalisation du processus d'enseignement-apprentissage des langues.

La question que l'enseignant-tuteur devrait se poser serait donc de savoir à quelle conception de la DLC initier ces étudiants pour ne pas trop simplifier les choses, mais en même temps ne pas risquer de les décourager à travers un discours glottodidactique, voire métaglottodidactique trop complexe et par conséquent incompréhensible pour les chercheurs novices. Ainsi, étant donné que le processus d'enseignement / apprentissage des langues - qui constitue l'objet des études de la DLC - est par excellence interdisciplinaire, l'enseignanttuteur devrait se demander comment sensibiliser ses étudiants à l'interdisciplinarité de la DLC. Et, si les chercheurs expérimentés sont obligés de traiter l'interdisciplinarité comme une pratique hautement complexe qui consiste en un dialogue entre spécialistes de différentes disciplines qui réorganisent leur encadrement théorique, mais aussi méthodologique, tout en prenant en considération la spécificité épistémologique de chaque discipline (cf. Wilczyńska \& Michońska-Stadnik 2010), il suffirait, à notre sens, que les étudiants de licence, qui s'initient à peine à la dimension épistémologique de la DLC, et notamment à son interdisciplinarité, considèrent l'objet de leur étude comme interdisciplinaire dans le sens qu'il est complexe et multidimensionnel, sans chercher à recourir à l'interdisciplinarité à travers les adaptations de nature méthodologique réservées plutôt aux chercheurs expérimentés. 


\section{DES ÉTAPES DANS LE DÉVELOPPEMENT DE LA CONSCIENCE ÉPISTÉMOLOGIQUE DES ÉTUDIANTS}

Il est temps maintenant de décrire les différentes étapes dans le développement de la conscience épistémologique des étudiants en ethnolinguistique telles que nous les voyons pour par la suite définir les articulations entre la prise de conscience par ces étudiants de la spécificité épistémologique de la DLC et leur parcours en tant qu'étudiants plurilingues, chercheurs en DLC et, éventuellement, aussi enseignants de langues. Puis, nous chercherons à démontrer quelles démarches formatives concrètes pourraient être appliquées par le tuteur du ML lorsque ses étudiants en $3^{e}$ année de licence le consultent, tout au long de la préparation de leur premier écrit de recherche et donc à l'étape de leur entrée en écriture de recherche en glottodidactique.

\subsection{Vers une vision cohérente de la progression en matière de conscience épistémologique}

Pour pouvoir nous prononcer sur la progression en matière de conscience épistémologique des étudiants polonais en ethnolinguistique, nous sommes obligée de tenir compte de la spécificité de leur formation universitaire. Or, le principal trait distinctif des études universitaires en ethnolinguistique est celui de former les étudiants plurilingues qui de surcroît sont focalisés sur le développement de leur compétence de communication personnalisée et leur compétence d'apprentissage plurilingue (cf. Wilczyńska 2002, 2004), et non sur leur habilité professionnelle à enseigner. Par conséquent, ce qui est crucial à long terme pour ces étudiants, c'est de développer non seulement leur conscience disciplinaire, si fonctionnelle dans le cas des (futurs) enseignants de langues (Orchowska 2008a, 2008b, 2009, 2010a, 2010b ; Karpińska-Musiał \& Orchowska 2014), mais aussi et surtout, leur conscience épistémologique qui devrait interagir avec leur compétence d'apprentissage plurilingue.

Autrement dit, nous considérons comme essentielle dans la formation universitaire de nos étudiants leur prise de conscience par rapport à la spécificité épistémologique des connaissances du domaine de la DLC en tant que science empirique dont l'objet de recherche s'inscrit dans le contexte réel, tout en nécessitant sa modélisation scientifique. Ainsi, dans le tableau ci-dessous, nous proposons notre vision de la progression en matière de développement de la conscience épistémologique des étudiants, en prenant en considération leur parcours formatif du statut d'un étudiant plurilingue de philologie au niveau de la licence jusqu'à celui d'un jeune chercheur en DLC qui est en train de s'intégrer à la communauté didactologique, tant sur le plan scientifique que discursif : 
Tableau 1. Étapes du développement de la conscience épistémologique des étudiants polonais en ethnolinguistique

\begin{tabular}{|c|c|}
\hline $\begin{array}{l}\text { Les étapes } \\
\text { de la formation } \\
\text { universitaire }\end{array}$ & $\begin{array}{l}\text { Les types de démarches didactiques appliquées en vue de développer } \\
\text { la conscience épistémologique des étudiants }\end{array}$ \\
\hline & 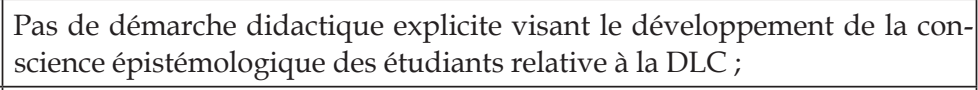 \\
\hline $\begin{array}{l}\text { 2ème année } \\
\text { d'études } \\
\text { supérieures }\end{array}$ & $\begin{array}{l}\text { démique et science empirique dont l'objet d'étu } \\
\text { interdisciplinaire à travers le cours facultatif d }\end{array}$ \\
\hline $\begin{array}{l}\text { 3ème année } \\
\text { d'études } \\
\text { supérieures } \\
\text { (le mémoire de } \\
\text { licence) }\end{array}$ & $\begin{array}{l}\text { Modalité pédagogique particulière d'accompagnement à l'entrée explicite } \\
\text { dans le domaine de la DLC, y compris en écriture de recherche glottodidac- } \\
\text { tique, sous forme de consultations régulières entre les étudiants et leur tutrice } \\
\text { et de démarches d'écriture et de reécriture du texte de leur ML, objet de } \\
\text { l'évaluation et de l'auto-évaluation favorisant ainsi l'émergence et le dével- } \\
\text { oppement explicite et autonome de la conscience épistémologique réflexive ; }\end{array}$ \\
\hline $\begin{array}{l}1^{\text {ère }} \text { et } 2^{\text {ème }} \text { année de } \\
\text { master } \\
\text { (le mémoire de }\end{array}$ & $\begin{array}{l}\text { dente, } \\
\text { s dans }\end{array}$ \\
\hline $\begin{array}{l}\text { Études c } \\
\text { (la thèse } \\
\text { doctorat }\end{array}$ & $\begin{array}{l}\text { ultations visant l'auto-formation à la recherche } \\
\text { isciplinaire. }\end{array}$ \\
\hline
\end{tabular}

Comme le tableau ci-dessous le montre, la progression en matière de développement de la conscience épistémologique de nos étudiants devrait se réaliser à travers le passage de la domination de doxa à la suprématie de l'épistémè dans leurs représentations relatives à l'enseignement-apprentissage des langues (cf. Narcy-Combes 2010), et donc par leur rupture épistémologique avec les idées qu'ils s'étaient créées avant d'être initités à ce qu'est la DLC en tant que domaine de recherche qui constitue une science empirique aspirant à l'autonomie cognitive (cf. Dakowska 2014). Ainsi, il s'agit, tout au long du cursus en ethnolinguistique, $\mathrm{d}$ 'amener les chercheurs novices vers l'autonomie en matière d'appropriation du discours et des savoirs qui relèvent des recherches menées sur le terrain de la glottodidactique. Et au niveau des études doctorales, il s'agit même de les préparer à l'élaboration de leurs savoirs transversaux, car leur conscience épistémologique devrait aussi leur permettre de dépasser les frontières disciplinaires et s' ouvrir à l'interdisciplinarité des sciences sociales et humaines, tout en reconnaissant la spécificité épistémologique de leur discipline de spécialité. 
Quant aux trois premières années d'études universitaires, l'enjeu majeur de la formation sur le plan épistémologique est de conduire les étudiants d'ethnolinguistique à devenir des apprenants de langues avertis qui sont à même de recourir aux connaissances glottodidactiques pour mieux gérer leur apprentissage personnel, tout en reconnaissant le caractère pluriligue et pluriculturel de leur compétence de communication, mais aussi pour mener une réflexion didactologique sur les représentations d'autres acteurs de l'enseignement-apprentissage des langues (et cultures) ou bien effectuer une analyse critique du contenu des dispositifs d'enseignement-apprentissage élaborés par les autres et disponibles en version papier ou sur Internet. Nous sommes persuadée que les étudiants - grâce à l'émergence de leur concience épistémologique réflexive peuvent s'épanouir de façon plus autonome et efficace aussi bien au niveau de leur compétence de communication personnalisée qu'à celui de leur compétence d'apprentissage. En effet, ils sont censés se rendre compte quelles démarches $d^{\prime}$ 'enseignement et quelles stratégies d'apprentissage correspondent le mieux au développement de leur compétence de communication en langues-cultures cibles et pourquoi. Ainsi, ils deviennent des acteurs de leur apprentissage qui savent dialoguer avec leurs enseignants mais aussi effectuer une sélection consciente de démarches et stratégies à appliquer pour améliorer leurs compétences.

De plus, en troisième année de licence, les étudiants d'ethnolinguistique sont initiés à la recherche dans le domaine de la DLC, mais à cette étape de la formation universitaire on se focalise sur les recherches qualitatives et contextualisées et on privilégie la méthode de recherche telle que l'analyse qualitative de contenu. Par conséquent, c' est seulement à partir de la $1^{\text {ère }}$ année d'études en master que la formation de nos étudiants devrait inclure le développement leur conscience méthodologique à proprement parler. Enfin, à l'étape des études doctorales, réservée par ailleurs à un nombre très restreint de diplômés en philologies, non seulement on continue de développer leur conscience épistémologique disciplinaire, mais en outre on les ouvre déjà à un dialogue de recherche à caractère interdisciplinaire, ce qui d'ailleurs est nécessaire en raison la complexité de l'objet de recherche en DLC (cf. Puren 2000 ; Orchowska 2020).

\subsection{Comment le tuteur du ML en DLC devrait-il encadrer les étudiants?}

Étant donné la spécificité du contexte universitaire dans lequel sont formés les étudiants polonais d'ethnolinguistique, ce qui paraît essentiel dans le rôle à jouer par le tuteur de leur ML est sa façon de les encadrer épistémologiquement tout au long de leur troisième année d'études, notamment au niveau de l'organisation 
de leur travail de recherche et de rédaction ainsi que pendant les consultations qui constituent la forme de communication et d'enseignement principale lors de la rédaction par eux de leur premier écrit de recherche.

Ainsi, il serait maintenant pertinent d'expliquer quelles démarches formatives sont à entreprendre par le tuteur en vue de favoriser le développement de la conscience épistémologique des étudiants. Premièrement, à notre sens, le tuteur devrait proposer à ses étudiants un calendrier précis avec les objectifs à atteindre progressivement pendant la préparation de leur ML. En fait, c'est à l'enseignant-tuteur de faire comprendre à ses étudiants en quoi consiste la complexité du processus de préparation du ML en DLC et ce calendrier devrait comprendre tous les enjeux à relever à cette étape de la formation universitaire, tant sur le plan épistémologique que discursif. Ainsi, le calendrier en question devrait comprendre la réalisation des objectifs particuliers qui mènent l'étudiant vers la rédaction de son ML à partir de la préparation de la première version de son projet de recherche jusqu' à la version finale et intégrale de son premier écrit de recherche qu'est le ML en DLC (cf. Orchowska 2010a).

Puis, en ce qui concerne les consultations, les démarches à entreprendre par le tuteur devraient aider chaque étudiant à réaliser les objectifs formatifs suivants :

1) sélectionner une / des théorie/s de référence pour nommer, encadrer épistémologiquement et interpréter le problème didactologique qui sera approfondi dans le ML, y compris sélectionner progressivement les publications spécialisées à inclure sans la bibliographie et la réseaugraphie ;

2) sélectionner et définir les notions clés par rapport au problème analysé ;

3) expliquer les rapports entre différents aspects du problème analysé et les facteurs relatifs à sa meilleure compréhenion à caractère scientifique ;

4) présenter une vision d'ensemble du problème choisi, en précisant les contenus et les visées de différentes composantes du mémoire ;

5) expliquer et justifier ses choix épistémologiques, leur impact sur la terminologie utilisée et la méthodologie de recherche appliquée ;

6) faire dialoguer différents experts qui se sont déjà prononcés sur la question traitée à travers la rédaction du ML ;

7) réaliser une recherche didactologique qualitative qui illustrera empiriquement les idées présentées dans les chaîtres théoriques du ML ;

8) rédiger compte-rendu de sa recherche, en présenter les conclusions et ainsi que les limites de la démarche de recherche appliquée et, éventuellement, de nouvelles perspectives de recherche ;

9) après la lecture de la version intégrale de son ML, rédiger la version finale de son introduction et passer par la suite à la rédaction du résumé de l'ensemble de cet écrit de recherche en français (ou en polonais) et en anglais. 
L'énumération des objectifs à réaliser tout au long de la préparation du ML par nos étudiants pendant la troisième année de leurs études universitaires démontre bien que les connaissances visées à cette étape de leur formation ne se limitent pas à un savoir que, mais que les diplômés de licence en ethnolinguistique sont censés savoir sélectionner, contextualiser et définir un problème didactologique ainsi que justifier leurs choix épistémologiques et en prévoir les conséquences. Bref, on s'attend à ce qu'ils pensent de façon créative, critique et conforme aux principes épistémologiques de leur discipline de référence et qu'ils soient à même de rédiger leur $\mathrm{ML}$, en recourant aux normes discursives relatives à la communication scientifique entre les spécialistes en DLC.

Pour continuer notre réflexion, il serait approprié de réfléchir également sur la compétence discursive du tuteur et ce qui conditionne son efficacité didactique lorsqu'il interagit pendant les consultations avec ses étudiants qui préparent leur $\mathrm{ML}$, tout en ayant un impact considérable sur la qualité de leur écriture glottodidactique. Il s'agira notamment de répondre à la question de savoir ce qui pourrait donner au feed-back de l'enseignant-tuteur le caractère dialogique qui rend l'échange communicatif entre les étudiants et leur formateur efficace, y compris par rapport à leurs littéracies universitaires et leur autonomie en tant que (futurs) chercheurs (cf. Orchowska 2010b). Certes, une fois de plus, cet échange devrait s'inscrire dans une communication entre les spécialistes en DLC qui prennent en considération et comprennent la spécificité épistémologique et discursive de leur discipline de spécialité. Autrement dit, à notre sens, l'émergence de la conscience épistémologique de nos étudiants constitue une étape dans leur (auto)formation philologique qui est incontournable, car cette prise de conscience leur facilite non seulement la rédaction des écrits de recherche, mais également l'appropriation des savoirs qui relèvent de leur discipline académique de spécialité, de même que le dialogue interdisciplinaire avec d'autres spécialistes en sciences humaines et sociales.

\section{EN GUISE DE CONCLUSION}

Ce qui précède nous amène à constater que ce qui est crucial sur le plan formatif dans l'initiation des étudiants en ethnolinguistique à l'écrit glottodidactique est le développement de leur conscience épistémologique aussi bien par rapport à la spécificité épistémologique de la DLC que pour ce qui est de la nature des sciences humaines et sociales en général. Dans ce dernier cas, on peut d'ailleurs parler des savoirs dits transversaux dont le développement devrait être privilégié avant tout au niveau des études doctorales.

En outre, nous postulons que la prise de conscience à caractère épistémologique de la part des étudiants soit accompagnée du développement de leur 
pensée critique dont la formation devrait constituer un objectif immanent de l'enseignement supérieur, notamment en Pologne où la formation de l'esprit critique des élèves fut négligée pendant des années en tant qu'objectif d'apprentissage dans le secondaire (cf. Orchowska 2008b). En effet, la pensée critique est indispensable pour s'approprier et appliquer de façon autonome ces savoirs disciplinaires et transversaux, mais il faut reconnaître que la pensée critique constitue une posture cognitive incontournable dans tout le processus d'enseignement-apprentissage des langues et cultures. Ainsi, d'après nous, elle devrait être considérée comme une composante de la compétence d'apprentissage plus générale des étudiants.

Nous pouvons clore notre réflexion sur le développement de la littéracie glottodidactique dans le cadre de la formation universitaire des étudiants polonais en ethnolinguistique en constatant que ceux-ci pourront progresser considérablement en matière $\mathrm{d}^{\prime}$ appropriation du discours glottodidactique et des savoirs diffusés à travers ce discours à condition qu'ils soient accompagnés lors de la rédaction de leurs ML en DLC par les démarches didactiques favorisant le développement de leur conscience épistémologique relative au fonctionnement de la glottodidactique en tant que discipline académique et domaine de recherche. En effet, ce qui est crucial dans l'accompagnement des étudiants lors de la rédaction de leur ML, c'est de les accompagner de telle façon qu'ils sachent sélectionner consciemment, contextualiser et encadrer épistémologiquement des problèmes didactologiques ainsi qu'ils soient conscients de l'impact de leur positionnement épistémologique et leur choix méthodologique sur la façon dont ils interprètent, décrivent et analysent le problème choisi.

Nous pouvons donc conclure que la réussite des étudiant(e)s dans l'appropriation de l'écrit glottodidactique est inséparable de la compréhension par ceux-ci de la spécificité épistémologique du domaine de recherche dans lequel leur écrit se situe. En effet, c'est grâce à cette prise de conscience à caractère épistémologique que la formation en question contribue au processus d'autonomisation des scripteurs, ce qui conditionne leur succès en tant que chercheurs novices, de même que leur autoformation aux stades plus avancés de leur parcours académique.

Il serait également juste de remarquer que les articulations entre la conscience épistémologique des étudiants en ethnolinguistique et leur futur professionnel en tant que chercheurs spécialisés en DLC paraissent incontestables aussi sur le plan communicatif, car la conscience épistémologique des glottodidacticiens et la maîtrise du discours spécialisé constituent la condition sine qua de leur participation active au dialogue au sein de la communauté didactologique ainsi qu'à la communication interdisciplinaire avec les représentants des sciences connexes. 


\section{RÉFÉRENCES}

Bachelard, G. (1938). La Formation de l'esprit scientifique. Contribution à une psychanalyse de la connaissance objective. Paris: Vrin.

Chiss, J.-L. (2010). La didactique des langues: une théorie d'ensemble et des variables ? In: D. Macaire / J. P. Narcy-Combes / H. Portine (dir.). Interrogations épistémologiques en didactique des langues (pp. 37-45). Paris: Clé International.

Chiss, J.-L. (2012). De la littératie aux littératies: conceptions et frontières. Recherches en didactique des langues et des cultures. Les Cahiers de l'Acedle, 9 (2), 1-5.

Dakowska, M. (2010). W poszukiwaniu wiedzy praktycznie użytecznej. O dojrzewaniu glottodydaktyki jako dziedziny akademickiej. Neofilolog, 34, 9-19.

Dakowska, M. (2014). O rozwoju dydaktyki języków obcych jako dyscypliny naukowej. Warszawa: Wydawnictwa Uniwersytetu Warszawskiego.

Dakowska, M. (2018). Professor Grucza as the founder of glottodidactics. Kwartalnik Neofilologiczny $L X V, 4,655-658$.

Delcambre, I. / Lahanier-Reuter, D. (2012). Littéracies universitaires: présentation. In: I. Delcambre / D. Lahanier-Reuter (dir.). «Littéracies universitaires: nouvelles perspectives», Pratiques. Linguistique, littérature, didactique, 3-19, 153-154.

Galisson, R. (1986). Éloge de la Didactologie / Didactique des Langues et des Cultures (maternelles et étrangères) - D/DLC. Études de Linguistique Appliquée, 64, 97-110.

Galisson, R. (1990). 1990. Où va la didactique du français langue étrangère ? In: R. Galisson (dir.). «De la linguistique appliquée à la didactologie des langues-cultures: vingt qns de réflexion», Études de Linguistique Appliquée. Revue de didactologie des langues-cultures, 79, 9-28.

Galisson, R. / Puren, Ch. (1999). La formation en question. Paris: CLE International.

Karpińska-Musiał, B. (2016). Edukacja spersonalizowana w uniwersytecie. Ideologia - instytucja - dydaktyka - tutor. Kraków: Wydawnictwo Libron.

Karpińska-Musiał, B. / Orchowska, I. (2014). Świadomość przedmiotowa i epistemologiczna nauczyciela - refleksyjnego praktyka z perspektywy polskiej glottodydaktyki. Neofilolog, 43/1, 25-38.

Narcy-Combes, J.-P. (2010). Illusion ontologique et pratique réflexive en didactique des langues. In: D. Macaire / J.-P. Narcy-Combes / Portine, H. (dir.). Interrogations épistémologiques en didactique des langues (pp. 111-122). Paris: Clé International.

Orchowska, I. (2008a). La formation interculturelle des futurs enseignants de FLE dans le contexte universitaire polonais. Kraków: Flair.

Orchowska, I. (2008b). Les pratiques discursives orales à visée argumentative et la perspective interculturelle en FLE. In: J. Lis / T. Tomaszkiewicz (dir.). Francophonie et interculturalité (pp. 123-130). Łask: Oficyna Wydawnicza Leksem.

Orchowska, I. (2009). Rozwijanie kompetencji dyskursywnej w KJO w zakresie dyskursu naukowego w języku francuskim jako obcym: wyzwania językowe, dyskursywne i interkulturowe. In: J. Nijakowska (dir.). Jezzyk - poznanie - zachowanie: Perspektywy i wyzwania w studiach nad przyswajaniem języka obcego (pp. 173-189). Łódź: Wydawnictwo Uniwersytetu Łódzkiego.

Orchowska, I. (2010a). Konstruowanie projektu pracy dyplomowej jako element kształcenia akademickiego przyszłych nauczycieli języków obcych. Neofilolog, 34, 111-122.

Orchowska, I. (2010b). Wpływ strategii dyskursywnych na realizację celów uczeniowych i heurystycznych w sytuacji konsultacji seminaryjnych. In: M. Pawlak / E. Waniek-Klimczak (dir.). Mówienie w języku obcym - sukcesy i porażki uczenia się i nauczania (pp. 313-327). Poznań - Kalisz - Konin: Wydawnictwo UAM / PWSZ w Koninie. 
Orchowska, I. (2013a). De la communication spécialisée à l'intérieur de la communauté glottodidactique polonaise. Conceptualisation de l'objet de la discipline scientifique et de son interdisciplinarité. In: E. Wąsikiewicz-Firlej / H. Lankiewicz (dir.). From classroom to workplace: Advances in applied linguistics (pp. 189-206). Piła: Wydawnictwo PWSZ im. S. Staszica w Pile.

Orchowska, I. (2013b). O rozbieżnościach konceptualnych w definiowaniu zakresu obszaru badawczego glottodydaktyki wewnątrz wspólnoty naukowej polskich glottodydaktyków. In: P. Nowakowski / K. Stroński / M. Szczyszek. (dir.). «Od wspólnot komunikatywnych do kontaktów międzywspólnotowych», Poznańskie Spotkania Językoznawcze, 26 (61-77). Poznań: Wydawnictwo Poznańskiego Towarzystwa Przyjaciół Nauk.

Orchowska, I. (2014a). De l'autonomie scientifique de la didactologie des langues étrangères en Pologne. L'analyse comparative des conceptions de trois épistémologues contemporains polonais de la discipline. In: J. I. Ignacio Aguilar Río / C. Brudermann / M. Leclère (dir.). Complexité, diversité et spécificité: Pratiques didactiques en contexte (pp. 181-195). Paris: Université Sorbonne Nouvelle - Paris 3. Repéré à: https:/ / halshs.archives-ouvertes.fr/halshs-01099170 / document [access: 11.03.2020].

Orchowska, I. (2014b). Rozwijanie glottodydaktycznej świadomości epistemologicznej przyszłych nauczycieli a sukces w procesie nauczania-uczenia się języka obcego. In: B. Karpeta-Peć / R. Kucharczyk / M. Smuk / M. Torenc (dir.). Wyznaczniki sukcesu nauczyciela i ucznia w glottodydaktyce (pp. 77-97). Warszawa: Instytut Germanistyki UW / Instytut Romanistyki UW.

Orchowska, I. (2017). De la spécificité épistémologique du discours méta-glottodidactique. Entre autonomie intellectuelle des chercheurs et leur appartenance à la communauté scientifique polonaise. Studia Romanica Posnaniensia, 44 (2), 27-45.

Orchowska, I. (2020). O autonomii przedmiotu poznania polskiej dydaktyki J2. Od syntezy do metaanalizy polskiego dyskursu metaglottodydaktycznego (en préparation).

Puren, Ch. (2000). Pour une didactique complexe. In: M. Marquillo Marruy (dir.). Les Cahiers FoReLLIS (Revue de la Maison de l'Homme et des Sociétés). Questions d'épistémologie en didactique du français (langue maternelle, langue seconde, langue étrangère), 16, 21-29.

Schön, D. 1983. The reflective practitioner. How professionals think in action. London: Temple Smith.

Wilczyńska, W. (1996). Mémoire de diplôme au NKJO. Petit guide. Poznań: Instytut Filologii Romańskiej.

Wilczyńska, W. (2002). Podmiotowość i autonomia jako wyznaczniki osobistej kompetencji komunikacyjnej. In: W. Wilczyńska (dir.). Autonomizacja w dydaktyce języków obcych. Doskonalenie się w komunikacji ustnej (pp. 51-67). Poznań: Wydawnictwo UAM.

Wilczyńska, W. (2004). Identité bilingue et compétence personnalisée. French Studies in Southern Africa, 33, 94-112.

Wilczyńska, W. (2010). Obszary badawcze glottodydaktyki. Neofilolog, 34, 21-35.

Wilczyńska, W. / Michońska-Stadnik, A. (2010). Metodologia badań w glottodydaktyce. Wprowadzenie. Kraków: Avalon.

Zając, J. (2010). Glottodydaktyka w ujęciu epistemologicznym. Neofilolog, 34, 37-48. 


\begin{abstract}
ANNEXES
Annexe 1

\section{Programme du cours facultatif d'introduction à la DLC Introduction à la didactologie des langues-cultures}

Cours facultatif sous forme de TD avec des éléments d'un cours magistral

Enseignante : Izabela Orchowska

Objectifs généraux du cours : s'initier aux dimensions épistémologique, pratique et (inter) culturelle de la didactologie des langues et cultures pour mieux gérer son apprentissage des langues

\title{
Questions qui seront abordées :
}

- Qu'est-ce que la didactologie des langues et cultures (son objet et son champ de recherche, son métalangage, sa spécificité épistémologique et méthodologique, ses subdisciplines)?

- Qu'est-ce qu'apprendre une langue, quels facteurs influencent-ils l'apprentissage et qu'est-ce qui conditionne son efficacité ?

- Qu'est-ce qu'une compétence de communication et une compétence d'apprentissage personnelles en L2 et comment développer ces compétences à différents niveaux de référence?

- Que signifie le passage de la didactique autonomisante à la perspective actionnelle et plurilingue du Cadre commun,

- En quoi consiste la spécificité de l'enseignement-apprentissage des langues par les personnes plurilingues?

- Qu'est-ce que l'approche interculturelle dans l'enseignement-apprentissage des langues?

- Comment exploiter les documents authentiques et recourir aux nouvelles technologies de l'information et de la communication dans l'enseignement-apprentissage des langues visant les compétences de communication et d'apprentissage personnelles des apprenants?

\section{Matériel et supports pédagogiques :}

- articles et extraits d'ouvrages spécialisés du domaine de la didactologie des langues et cultures,

- manuels / méthodes et enregistrements d'extraits de cours de langues pour servir d'exemples et/ou d'objets à l'analyse critique, chronologique et / ou comparative,

- documents authentiques (illustrations, photos, documents ethnographiques comme menus de restaurants, billets, cartes postales., extraits de films et d'émissions de radio et de télévision, enregistrements audio et transcriptions de paroles de chansons, etc.). 


\section{Conditions d'obtention de la signature ${ }^{7}$ :}

- ne pas dépasser deux absences non justifiées par semestre,

- participer activement aux cours (on peut être non préparé(e) au maximum deux fois par semestre et on doit le comuniquer avant le cours),

- réussir une discussion orale avec l'enseignante sur l'une des problématiques abordées (les règles précises de cette discussion seront présentées par l'enseignante pendant la deuxième séance du cours),

- préparer individuellement, à deux ou à trois un glossaire franco-polonais avec le langage (méta)glottodidactique et faire preuve de maîtrise de ce langage lors de l'entretien oral à la fin du semestre.

\section{RÉFÉERENCES}

Blanchet, Ph. / Coste, D. (2010). Regards critiques sur la notion d'interculturalité. Pour une didactique de la pluralité linguistique et culturelle. Paris: L'Harmatton.

Chłopek, Z. (2011). Nabywanie języków trzecich i kolejnych oraz wielojęzyczność. Aspekty psycholingwistyczne (i inne). Wrocław: Wydawnictwo Uniwersytetu Wrocławskiego.

Coste, D. / North, B. / Sheil, J. / Trim, J. (2001). Cadre européen commun de référence pour les langues: apprendre, enseigner, évaluer. Paris: Conseil de l'Europe / Didier.

Dakowska, M. (2003). Current controversies in foreign language didactics. Warszawa: Wydawnictwo Uniwersytetu Warszawskiego.

Dakowska, M. (2014). O rozwoju dydaktyki języków obcych jako dyscypliny naukowej. Warszawa: Wydawnictwo Uniwersytetu Warszawskiego.

Galisson, R. (1986). Éloge de la Didactologie / Didactique des Langues et des Cultures (maternelles et étrangères) - D/DLC. Études de Linguistique Appliquée, 64, 97-110.

Galisson, R. (1990). Où va la didactique du français langue étrangère ? In: R. Galisson (dir.). De la linguistique appliquée à la didactologie des langues-cultures: vingt qns de réflexion. Études de Linguistique Appliquée. Revue de didactologie des langues-cultures, 79, 9-28.

Galisson, R. (2002). Didactologie: de l'éducation aux langues-cultures à l'éducation par les langues-cultures. Études de Linguistique Appliquée, 128, 497-510.

Mangenot, F., Zourou, K. (2007). Susciter le dialogue interculturel en ligne. Rôle et limites des tâches. Lidil, 35, 43-68.

Nijakowska, J. (dir.). 2009. Perspektywy i wyzwania w studiach nad przyswajaniem języka obcego. Łódź: Wydawnictwo Uniwersytetu Łódzkiego.

North, B. / Goodier, T. / Piccardo, E. (2018). Cadre européen commun de référence pour les langues: apprendre, enseigner, évaluer. Volume complémentaire avec de nouveaux descripteurs. Conseil de l'Europe.

Orchowska, I. (2007). La formation interculturelle des futurs enseignants de FLE dans le contexte universitaire polonais. Kraków: Flair.

Orchowska, I. (2014a). Approche interculturelle et spécificité épistémologique de la didactologie des langues et cultures étrangères. In: E. Wąsikiewicz-Firlej / H. A. Lankiewicz / A. Szczepaniak-

${ }^{7}$ Les personnes intéressées pourront se présenter à l'examen oral ou écrit selon leur choix dont la date sera négociée et fixée avec l'enseignante. 
-Kozak (dir.). Culture and creativity in discourse studies and foreign language pedagogy (pp. 25-38). Piła: Wydawnictwo PWSZ im. S. Staszica w Pile.

Orchowska, I. (2014b). De l'autonomie scientifique de la didactologie des langues étrangères en

Pologne. L'analyse comparative des conceptions de trois épistémologues contemporains

polonais de la discipline. In: J. I. Ignacio Aguilar Río / C. Brudermann / M. Leclère (dir.). Complexité, diversité et spécificité: Pratiques didactiques en contexte (pp.181-195). Paris: Université

Sorbonne Nouvelle - Paris 3.

Valdman, A. (2003). The acquisition of sociostylistic and sociopragmatic variation by instructed second language learners: the elaboration of pedagogical norms. In: C. Blyth (dir.), The sociolinguistics of foreign-language classroom: Contribution of the native, the near-native, and the non-native speaker (pp. 57-78). Boston: Heinle.

Wilczyńska, W. (1999). Uczyć się czy być nauczanym? O autonomii w przyswajaniu języka obcego. Warszawa / Poznań: PWN.

Wilczyńska, W. (2010). Introduction à la didactique du FLE. Kraków: Flair.

Wilczyńska, W. / Michońska-Stadnik, A. (2010). Metodologia badań w glottodydaktyce. Wprowadzenie. Kraków: Flair.

\section{RÉSEAUGRAPHIE}

Blanchet, P. (2009). Contextualisation didactique: de quoi parle-t-on ?. Le français à l'université. http:/ / eprints.aidenligne-francais- universite.auf.org/147/1/pdf.pdf [access: 11.03.2020].

Charaudeau, P. (1988). L'interculturel, une histoire de fou. Dialogues et cultures 32. http:/ / www. patrick-charaudeau.com/L-interculturel-une-histoire-de,114.html [access: 11.03.2020].

Charaudeau, P. (2010). Pour une interdisciplinarité 'focalisée' dans les sciences humaines et sociales. Questions de communications. http:/ / questionsdecommunication.revues.org/385 [access: 11.03.2020].

\section{Annexe 2}

Liste des titres des ML en DLC rédigés entre octobre 2012 et septembre 2019 sous la tutelle de l'auteure du présent article par les étudiantes d'ethnolinguistiqueà l'Université Adam Mickiewicz de Poznań

Tableau 2. Liste des titres des ML en DLC rédigés entre octobre 2012 et septembre 2019 sous la tutelle de l'auteure du présent article par les étudiantes d'ethnolinguistique à l'Université Adam Mickiewicz de Poznań

Titres des ML en versions originales et leurs traductions en français entre parenthèses, si nécessaire

Rozwijanie kompetencji interkulturowej w nauczaniu-uczeniu się języka francuskiego jako obcego na przykładzie analizy treści podręcznika Déjà vu 1 (fr. Développer la compétence interculturelle pendant l'enseignement-apprentissage du FLE. Analyse du contenu du manuel Déjà vu 1) 
Sensibiliser les apprenants polonais de FLE aux différences et similitudes interculturelles dans la communication orale. Niveau B2

O świadomości metajęzykowej i metakognitywnej polskich studentów trójjęzycznych (fr. Sur la conscience métalinguistique et la conscience métacognitive des étudiants trilingues polonais)

Kompetencja interkulturowa $\mathrm{w}$ dydaktyce zadaniowej na przykładzie analizy podręcznika do języka francuskiego Nouveau Rond-Point 1 (fr. La compétence interculturelle dans la perspective actionnelle. Analyse du contenu du manuel Nouveau Rond-Point 1)

O wpływie inputu językowego na rozwój kompetencji leksykalnej i semantycznej dzieci dwujęzycznych. Studium przypadku (fr. L'impact de l'input linguistique sur le développement des compétences lexicale et sémantique des enfants bilingues. Étude de cas)

Du rôle de la littérature dans l'enseignement / apprentissage du français langue étrangère. Analyse qualitative du manuel Alter Ego 5

O rozwijaniu kompetencji komunikacyjnej w zakresie komunikacji ustnej u dzieci w wieku 6-8 lat. Jakościowa analiza treści podręcznika Les Loustics A1(fr. Développer la compétence de communication orale des enfants de 6 à 8 ans. Analyse qualitative du contenu du manuel Loustics A1).

La dimension (inter)culturelle dans l'enseignement-apprentissage du FLE. De la synthèse des principes didactologiques à l'analyse du contenu des manuels choisis.

Received: 29.08.2019; revised: 21.03.2020

\section{IZABELA ORCHOWSKA}

Uniwersytet im. Adama Mickiewicza w Poznaniu

izaorch@amu.edu.pl

ORCID: 0000-0003-0239-6160 
